\title{
BMJ Open Impact of public-funded health insurances in India on health care utilisation and financial risk protection: a systematic review
}

\author{
Bhageerathy Reshmi, ${ }^{1}$ Bhaskaran Unnikrishnan, ${ }^{2}$ Eti Rajwar, ${ }^{3}$ \\ Shradha S Parsekar (D) , ${ }^{3}$ Ratheebhai Vijayamma, ${ }^{4}$ Bhumika Tumkur Venkatesh (1) ${ }^{3}$
}

To cite: Reshmi B,

Unnikrishnan B, Rajwar E, et al. Impact of public-funded health insurances in India on health care utilisation and financial risk protection: a systematic review. BMJ Open 2021;11:e050077. doi:10.1136/ bmjopen-2021-050077

- Prepublication history and additional supplemental material for this paper are available online. To view these files, please visit the journal online (http://dx.doi.org/10.1136/ bmjopen-2021-050077).

Received 19 February 2021 Accepted 18 November 2021

Check for updates

(C) Author(s) (or their employer(s)) 2021. Re-use permitted under CC BY-NC. No commercial re-use. See rights and permissions. Published by BMJ.

For numbered affiliations see end of article.

Correspondence to

Dr Bhumika Tumkur Venkatesh; bhumika.tv@manipal.edu

\section{ABSTRACT}

Objective Universal Health Coverage aims to address the challenges posed by healthcare inequalities and inequities by increasing the accessibility and affordability of healthcare for the entire population. This review provides information related to impact of public-funded health insurance (PFHI) on financial risk protection and utilisation of healthcare.

Design Systematic review.

Data sources Medline (via PubMed, Web of Science), Scopus, Social Science Research Network and 3ie impact evaluation repository were searched from their inception until 15 July 2020, for English-language publications.

Eligibility criteria Studies giving information about the different PFHI in India, irrespective of population groups (above 18 years), were included. Crosssectional studies with comparison, impact evaluations, difference-in-difference design based on before and after implementation of the scheme, pre-post, experimental trials and quasi-randomised trials were eligible for inclusion.

Data extraction and synthesis Data extraction was performed by three reviewers independently. Due to heterogeneity in population and study design, statistical pooling was not possible; therefore, narrative synthesis was performed.

Outcomes Utilisation of healthcare, willingness-to-pay (WTP), out-of-pocket expenditure (including outpatient and inpatient), catastrophic health expenditure and impoverishment.

Results The impact of PFH on financial risk protection reports no conclusive evidence to suggest that the schemes had any impact on financial protection. The impact of PFHls such as Rashtriya Swasthy Bima Yojana, Vajpayee Arogyashree and Pradhan Mantri Jan Arogya Yojana showed increased access and utilisation of healthcare services. There is a lack of evidence to conclude on WTP an additional amount to the existing monthly financial contribution.

Conclusion Different central and state PFHls increased the utilisation of healthcare services by the beneficiaries, but there was no conclusive evidence for reduction in financial risk protection of the beneficiaries.

Registration Not registered.
Strengths and limitations of this study

- Inclusion of all kinds of empirical evidence to answer the research question about impact of public-funded health insurance (PFHI) schemes in India.

- This is one of the very few reviews that has used a systematic methodology to provide latest evidence on the impact of the newly launched Pradhan Mantri Jan Arogya Yojana scheme in India.

- Choice of quality appraisal tool, due to unavailability of other tools for this kind of study, was a limitation.

- Multiple PFHI (state-specific and central) schemes in India (with different benefit packages) and modifications in the schemes due to changes in central/state governments led to high data heterogeneity.

- Due to heterogeneity in data, we could not provide the pooled estimate via meta-analysis. However, results were explained via a narrative synthesis.

\section{INTRODUCTION}

India has a complex and mixed healthcare framework with presence of parallel public and private healthcare systems. ${ }^{12}$ There is a stark difference in government spending on both public and private healthcare. ${ }^{3}$ Health policies in India have been guided by the principle of equity with prioritising the needs of the poor and underprivileged. ${ }^{4}$ Out-ofpocket expenditure (OOPE) for health is one of the important factors while addressing the inequities in healthcare, and in India, it is an important source of healthcare financing. It is estimated that, in India, around $71 \%$ of the healthcare spending is met by OOPE. This not only is an immediate financial burden to the poor households but also pushes the households into a never-ending poverty trap. ${ }^{5}$ Health-related OOPE poses a threat to the principle of financial risk protection and adds to the unaffordability and inaccessibility of healthcare for the poor. High OOPE also leads to catastrophic health expenditure 
(CHE), which is the increase in healthcare payment by a household, beyond the threshold, where the threshold is defined as the household's income or capacity to pay. This is further divided into catastrophe 1, where healthcare OOPE exceeds by $10 \%$ of the household's consumption expenditure, and catastrophe 2, if OOPE exceeds to more than $40 \%$ of the household's non-food expenditure. The increase in OOPE affects the rural population marginally more than the urban population and the effect of OOPE is more pronounced among the people living below the poverty line (BPL) than those above the poverty line (APL), as BPL people are pushed more into poverty than APL, due to the high OOPE, when measured via the increase in poverty head counts. ${ }^{5}$

Over the years, government of India has rolled out different initiatives to address the healthcare-related inequities in India. The public healthcare system was revised and reframed as the National Rural Health Mission in 2005, later restructured as National Health Mission in 2014. ${ }^{56}$ Other initiatives like Janani Suraksha Yojana and the public funded health insurance (PFHI) schemes such as Rashtriya Swasthya Bima Yojana (RSBY) were also introduced to address the health inequalities, improve health outcomes and provide financial risk protection. ${ }^{6}$ Many states sponsored health insurance (HI) schemes, viz., the Vajpayee Arogyashree Scheme (VAS) by Karnataka, Comprehensive Health Insurance Scheme (CHIS) by Kerala and Chief Minister Health Insurance Scheme (CMHIS) by Tamil Nadu, which have been introduced for ensuring financial protection of the vulnerable population.

Challenges posed by healthcare inequalities and inequities like OOPE can also be addressed via the Universal Health Coverage (UHC). The UHC, as defined by the WHO, means that all people and communities can use the promotive, preventive, curative, rehabilitative and palliative health services they need, of sufficient quality to be effective, while also ensuring that the use of these services does not expose the user to financial hardship. The UHC aims towards increasing the accessibility and affordability of healthcare for the entire population. The definition of UHC is embodied in its three objectives, that is, equity, quality and financial protection. ${ }^{7}$

The twelfth 5-year plan of the government of India acknowledges the importance of UHC as it introduces a work plan for achieving UHC for the 1.3 billion population of the country. The agenda for this plan is based on the principle of providing affordable, accessible and good quality healthcare with financial protection to the people of the country. ${ }^{8}$ The provision of UHC has been included in the National Health Policy of India (2017). To achieve the UHC, government of India announced the 'Ayushman Bharat' programme in 2018 with two initiatives, that is, (a) Health and Wellness center and (b) National health protection scheme-Pradhan Mantri Jan Arogya Yojana (PMJAY), that is intended to cover around 500 million beneficiaries (from vulnerable families) and is intended to cover up to Indian National Rupees (INR)
500000 per family, per year, for secondary and tertiary hospitalisation. ${ }^{9}$

The addition of PMJAY scheme to the various existing PFHI (central and state) schemes aims to increase the UHC, by increasing the affordability and accessibility of good quality healthcare. It is important to assess whether these schemes (including PMJAY) have been proven to be effective in improving health outcomes and providing financial protection to the vulnerable population. Following the principles of UHC, willingness to pay (WTP) for a particular HI scheme can also be used as an indicator to assess the affordability and effectiveness of a scheme in providing good quality healthcare. Additionally, data on beneficiaries willing to pay more or contribute more for a HI scheme (viz., CGHS) indirectly provide information on their satisfaction with the services provided by the scheme, therefore, making it an indicator to assess effectiveness of the scheme. The previous systematic review ${ }^{10}$ on assessing the effectiveness of PFHI schemes in India was conducted before complete rolling out of the PMJAY and, therefore, did not include findings on the effectiveness of the scheme (PMJAY). Also, this review ${ }^{10}$ did not provide information on the WTP component of assessing impact of the HI schemes. The present review was, therefore, conducted with an aim to provide information related to effectiveness of the central and statefunded HI schemes (including the PMJAY scheme) via healthcare utilisation, WTP and financial risk protection of the beneficiaries. This review was planned to answer the following research question: (a) What is the impact of PFHI schemes on access and utilisation of healthcare, willingness-to-pay and financial risk protection in India?

\section{METHODS}

This systematic review follows the methodology by Cochrane handbook for systematic review of interventions ${ }^{11}$ and the Preferred Reporting Items for Systematic Reviews and Meta-Analyses checklist was used to report the review. ${ }^{12}$

\section{Criteria for including studies in the review}

1. Population: population group above 18 years of age enrolled in a PFHI scheme in India.

2. Intervention: HI schemes funded by either central or state government, and that covers, range of services such as hospitalisation, out-patient charges, medicine costs, treatment procedures, etc. Different PFHI schemes in India, for example, RSBY, VAS, CMHIS and PMJAY were eligible to be included. Private or community-based HIs were not eligible to be included. Mixture of HIs was excluded provided a study carried out subgroup analysis for PFHIs.

3. Comparison: comparison group comprises of people who did not receive any PFHI services.

4. Outcomes: this review includes the following outcomes: (a) utilisation of healthcare, (b) WTP, (c) fi- 
nancial risk protection measured in terms of OOPE, CHE and impoverishment.

5. Study design: cross-sectional studies with comparison, impact evaluations, difference-in-differences design based on before and after implementation of the scheme, pre-post design, experimental trials and quasi-randomised trials were eligible to be included.

\section{Search methods for identification of studies}

Electronic databases such as Medline (via PubMed, Web of Science), SCOPUS, Social Science Research Network and International Initiative for impact evaluation (3ie) repository were searched from their inception until 15 July 2020; however, only English publications, published in the last 10 years were considered. References and forward citations of the included studies were scanned through for any additional eligible studies. Keywords were identified before the initiation of the search. The initial search was carried out in PubMed (online supplemental file 1) and was replicated in other databases. Search was conducted by a designated information scientist.

\section{Data collection}

Result of search strategy was imported to Endnote V.X7 reference manager software. Duplicates were removed and the unique citations were exported to Microsoft Excel spreadsheet for screening.

\section{Selection of studies}

Unique citations were subjected to title and abstract screening independently by two reviewers. Eligible abstracts of all the relevant studies as per the inclusion criteria were included for full-text screening (by BTV, ER and SSP) and relevant ones from these were included for analysis. Before initiating full-text screening, we tried to retrieve the full-text articles by contacting authors of the respective articles and the full texts that were not retrieved were excluded. Disagreements were resolved by discussion or by a third reviewer.

\section{Data extraction}

Data extraction was done (by ER, BTV, SSP) using a predesigned data extraction form. Information on variables such as bibliographic details (author names, publication year, journal name); study details (information about the objectives of the study and research question addressed); study setting (name of the state, rural/urban); participant characteristics (age, gender, socioeconomic status, occupation); intervention details (name and type of HI, mode of delivery of the HI, incentives given, healthcare services covered, time duration of seeking HI, any additional HIs); comparison details; outcome details (information about changes in accessibility of healthcare, utilisation of healthcare services, OOPE, WTP, health outcomes like morbidity and mortality, measurement of the outcomes, method used for measurement, time at which the outcome was measured) and study design details (type of study design and analysis) were extracted.
After pilot testing of the data extraction form, it was revised according to the modifications suggested by the team. Disagreements among the reviewers, during data extraction, were resolved by consensus, if still not resolved, third reviewer was approached for resolving the disagreements. Extracted data from all the included studies were cross-checked and independent extraction was done for one-third randomly selected studies.

\section{Methodological quality}

The methodological quality of the included studies was assessed using Effective Public Health Practice Project Quality Assessment Tool (EPHPP). ${ }^{13}$ This tool assesses methodological quality of the quantitative studies based on questions under the following seven domains, that is, (a) selection bias, (b) study design, (c) confounders, (d) blinding, (e) data collection method, (f) withdrawals and dropouts, $(\mathrm{g})$ intervention integrity and $(\mathrm{h})$ analysis. Quality assessment using this scale was performed independently by reviewers in groups of two. After discussion, global rating for the scale was followed and studies were marked as (1) methodologically strong, if none of the domains had any weak rating, (2) moderate, if at least one domain was marked as weak and (3) weak, if two or more domains were marked as weak. Quality assessment was performed using Microsoft excel spreadsheet.

\section{Data analysis}

Due to heterogeneity in data, narrative synthesis was performed to answer the research question. The results are summarised based on outcomes and types of PFHIs. The effect measures of included studies such as mean difference or correlation coefficients with appropriate CI and/or $\mathrm{p}$ values are reported.

\section{Public and patient involvement}

We did not involve public or patient during the process of this review.

\section{RESULTS}

The literature search on electronic databases generated 555 citation yield, out of which 179 were duplicates. Additionally, 17 records were identified from forward and backward reference checking. After title and abstract screening of 393 citations, 157 were included for full-text screening, of which finally 25 articles were included for data synthesis. Schematic representation of the selection process is shown in figure 1.

\section{Characteristics of included studies}

The summary of study characteristics is given in table 1 and the detailed characteristics of included studies are given in online supplemental file 1.

Impact of PFHI on financial risk protection, utilisation of healthcare and WTP

This systematic review provides evidence on the impact of different PFHI schemes that have been operational 


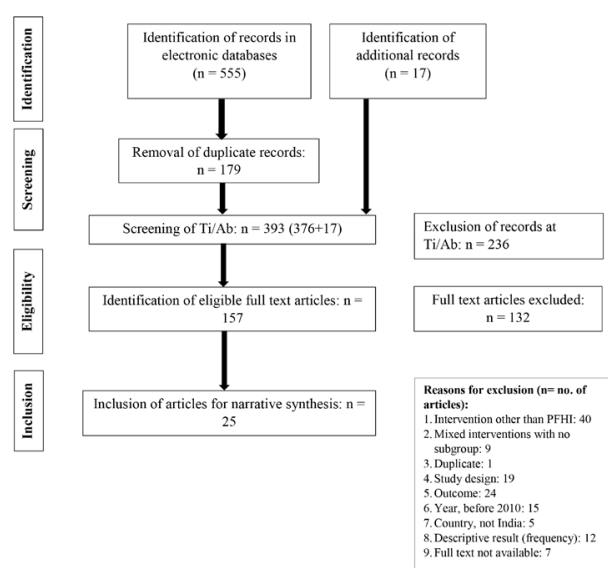

Figure 1 PRISMA flow diagram. PFHI, public-funded health insurance; PRISMA, Preferred Reporting Items for Systematic Reviews and Meta-Analyses.

in India. These schemes are funded by the central government, viz., RSBY, CGHS, Employee State Insurance Scheme, Swavlamban, Nirmaya-Disability Health Insurance Scheme and PMJAY and by the state governments like VAS (Karnataka), Rajiv Arogya Shree (Andhra Pradesh) and CHIS (Tamil Nadu). The eligibility criteria and benefits offered under each scheme vary according to different state governments. More information on these PFHI schemes is given in box 1 .

Summary of the impact findings of RSBY and other PFHIs is given in tables 2 and 3, respectively, and the detailed synthesis is provided in online supplemental file 1 .

\section{Financial risk protection}

Twenty-one studies measured financial risk protection, of which 17 were of strong methodological quality, ${ }^{14-30} 3$ of moderate methodological quality ${ }^{3-33}$ and 1 weak methodological quality. ${ }^{34}$ Nine studies ${ }^{141618192325303234}$ reported the impact of RSBY alone on financial protection. Thirteen studies ${ }^{15} 17$ 20-22 24 26-29 31-33 provided information on the effect of different PFHI schemes (including state insurance schemes) on financial risk protection.

Three high methodological quality studies reported a reduction in in-patient OOPE for RSBY households; ${ }^{14} 1830$ however, the findings were not significant. One low methodological study stated that after implementation of RSBY in Maharashtra state, there was a significant increase in in-patient expenditure for both public and private healthcare. ${ }^{32}$ RSBY did not have a significant effect on in-patient OOPE as a share of total health expenditure, this was reported by two good methodological studies. ${ }^{16}{ }^{19}$ The findings for the impact

\begin{tabular}{|c|c|c|}
\hline $\begin{array}{l}\text { Serial } \\
\text { number }\end{array}$ & $\begin{array}{l}\text { Study } \\
\text { characteristic }\end{array}$ & Summary \\
\hline 4. & Study design & $\begin{array}{l}\text { Impact evaluation including quasi-randomised designs was used in eight studies. }{ }^{1516192930323637} \text { Observational study } \\
\text { design was used in five studies. }{ }^{2325313338} \text { Secondary data analysis was performed in } 11 \text { studies. }{ }^{14171820-222426-2835} \text { Mixed } \\
\text { method approach was used in one study. }{ }^{34}\end{array}$ \\
\hline 5. & Outcomes & 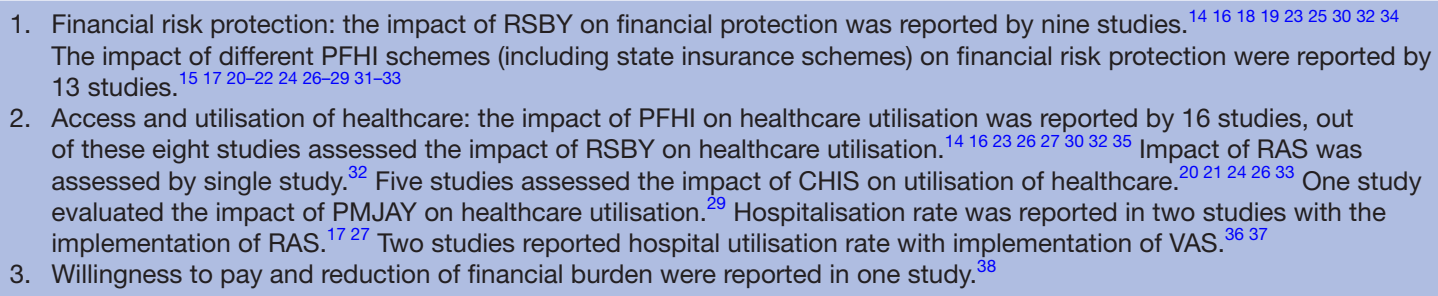 \\
\hline
\end{tabular}




\section{Box 1 Central and state-sponsored PFHI schemes in India}

\section{Central-funded health insurance schemes}

1. Rashtriya Swasthya Bima Yojana-RSBY (2008) is a central-funded health insurance scheme in which $75 \%$ of the annual premium is provided by the central government and rest $25 \%$ by the state governments. In-patient expenditure of upto Indian National Rupees (INR) 30000 per family per annum is insured for below poverty line families. Unorganised sector is also covered under this scheme.

2. Prime Minister's Jan Arogya Yojana-PMJAY (2018) is a fully government sponsored scheme, which provides a cover of INR 500000 per family per year in government empanelled public and private hospitals of India, for secondary and tertiary-level hospitalisation. Vulnerable and below the poverty line (BPL) families are eligible to avail the services under this scheme.

3. Central Government Health Scheme (1954) is eligible for central government employees and pensioners enrolled under the scheme. According to this scheme, inpatient services at the government empanelled hospitals, outpatient services including medicines, consultation by experts, maternity and child health services (family welfare) and medical consultation for alternative system of medicines are covered.

4. Swavlamban (2015), this is a central-funded health insurance scheme for people with disabilities. Eligible population includes BPL and differently abled people with blindness, hearing impairment, leprosy-cured, locomotor disability, mental illness, etc. A sum of INR 200000 per annum is covered and treatment of pre-existing illness is covered under the scheme.

5. Nirmaya-Disability Health Insurance Scheme (2008), this centralfunded health insurance scheme is specifically for people with Cerebral Palsy, autism, multiple disabilities and mental retardation. Services of upto INR 100000 are covered under this scheme.

6. Employee State insurance Scheme-Employee State Insurance Scheme(1952), this scheme is funded by the employers and staff contributions and is applicable to employees of factories and establishments drawing wages upto INR 15000 a month. Under this scheme, a number of benefits to protect the employees or workers from illness, disability and death are paid to the beneficiaries. Benefits such as sickness benefit (70\% of wages), temporary disablement benefit ( $90 \%$ of last wage), permanent disability benefit ( $90 \%$ of wage), maternity benefit ( $100 \%$ of wage), dependent benefit $(90 \%$ of wage), INR 10000 to dependents for funeral expenses in case of death of the employees and other benefits like vocational and physical rehabilitation are given to the beneficiaries.

State government-funded health insurance schemes

1. Aarogyasri Scheme (2007), this scheme is by the Telangana state and BPL families belonging to the state are eligible. Benefits include cashless transactions for treatment of extreme illness, for up to INR 200000 per year, covered under the scheme.

2. Ayushman Bharat-Mahatma Gandhi Rajasthan Swasthya Bima Yojana (2019), this scheme is by the government of Rajasthan and is formed by merging PMJAY scheme and Bhamashah Swasthya Bima Yojana. All the Rajasthani families belonging to BPL category are covered under this scheme. Under this scheme, an insured amount of INR 50000 and INR 450000 are provided for secondary and tertiary illness, respectively.

3. Chief Minister's Comprehensive Health Insurance Scheme (2012), this is a state-funded $\mathrm{HI}$ scheme by government of Tamil Nadu. People belonging to families of less than INR 72000 are annual earning or less and members of unorganised labour welfare boards,

Continued

\section{Box 1 Continued}

including their families are eligible. Services and benefits of up to INR 500000 per family per year are covered under the scheme.

4. Deen Dayal Swasthaya Seva Yojana (2016), by Goa government, for residents of Goa (residing for at least 5 years), central and state government employees already covered under other government health insurance benefits are eligible. Benefits include cashless inpatient services under government empanelled services. Annual coverage of upto INR 250000 for a family of three and INR 400000 for a family of four or more is given. Beneficiaries have to provide an annual premium of INR 200-300 to avail the benefits of the scheme.

5. Dr YSR Aarogyasri Scheme (Formerly called Rajiv Arogyasri Community Health Insurance Scheme)-2007, by the Andhra Pradesh government, this scheme covers BPL families from Andhra Pradesh. Under this scheme, free end-to-end cashless services are provided for patients undergoing treatment for therapies listed by the network hospitals. Free outpatient assessments are done for patients not undergoing treatment under the sited therapies.

6. Vajpayee Arogaya Shree (2009), this scheme is funded by the government of Karnataka and is applicable for BPL families from rural and urban areas of Karnataka. A total of INR 150000 is reimbursed for services provided to five members of the beneficiary family, an extra sum of INR 50000 per annum is provided in case-to-case basis.

7. West Bengal Health for All Employees and Pensioners Cashless Medical Treatment Scheme (2014), previously known as 'West Bengal Health Scheme', by the government of West Bengal, this scheme is for West Bengal government employees, pensioners and their family members. Benefits include reimbursement for in-patient services in the state empaneled hospitals and outpatient services for 15 diseases mentioned in the scheme. Cashless medical treatment for up to INR 100000 is provided for inpatient treatment.

8. Yeshasvini co-operative farmer's healthcare scheme (2003), by government of Karnataka, this scheme is for farmers who are members of the cooperative societies. According to this scheme, beneficiaries from the rural areas have to contribute INR 250 (for general category) and INR 50 (for SC/ST families) per annum. Beneficiaries from the urban areas have to contribute INR 710 (for general category) and INR 110 (for SC/ST) per annum. Benefits include inpatient services, discount rates for lab investigations, tests, outpatient services and medical emergency services due to mishaps during farming or any other agriculture related work.

of RSBY on outpatient OOPE were mixed as out of five good methodological quality studies, two studies mentioned that RSBY led to a reduction in outpatient OOPE, ${ }^{14} 18$ two studies reported that RSBY did not have any impact on the outpatient $\mathrm{OOPE}^{1630}$ and one study reported that the probability of incurring increased after implementation of RSBY. ${ }^{19}$ It was reported that the RSBY households were less likely to incur $\mathrm{CHE}$ for outpatient care, in-patient care and overall $\mathrm{CHE} ;{ }^{141619}$ however, one high methodological quality study reported that there was no impact of RSBY on CHE. ${ }^{25}$ All these findings were non-significant. The effect of RSBY on impoverishment was not clear as one study reported that RSBY had no effect on impoversihment, ${ }^{16}$ whereas another study reported an increase in impoverishment among the Above Poverty Line (APL) housholds. ${ }^{25}$ 
Table 2 Impact of RSBY on financial risk protection and healthcare utilisation

\begin{tabular}{|c|c|}
\hline Outcome & Findings \\
\hline $\begin{array}{l}\text { Out of Pocket health } \\
\text { Expenditure (OOPE) }\end{array}$ & 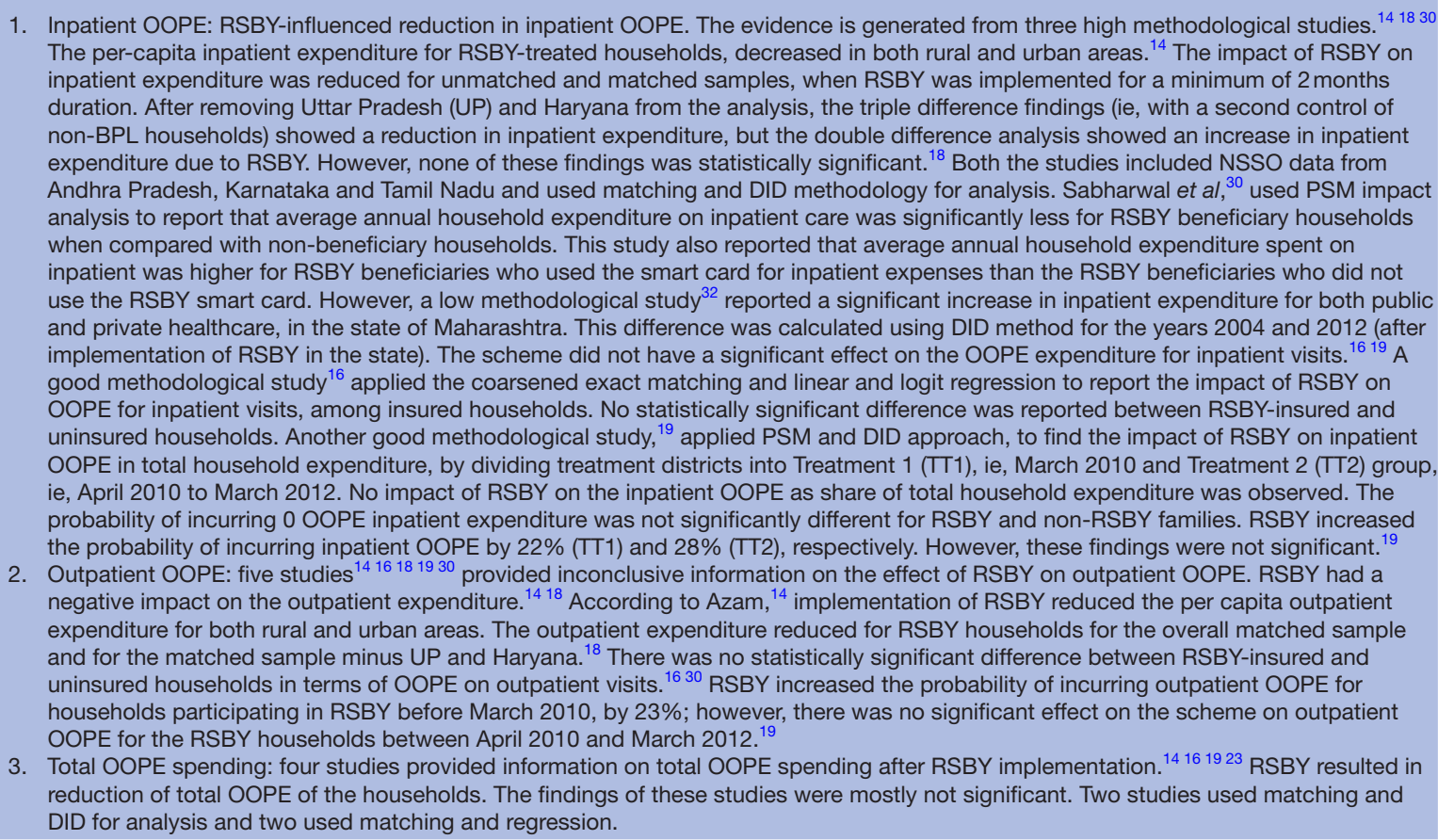 \\
\hline $\begin{array}{l}\text { alth } \\
\text { E) }\end{array}$ & $\begin{array}{l}\text { Four studies }{ }^{14161925} \text { provided information on the effect of RSBY on CHE, the RSBY households were less likely to incur CHE for outpatient } \\
\text { care, inpatient care and overall CHE. It was observed that beneficiaries of the scheme reported a reduction in CHE; however, one study } y^{25} \\
\text { reported that there was no effect of RSBY on CHE. According to Azam, }{ }^{14} \text { the effect was same for both rural and urban households. RSBY } \\
\text { increased the likelihood of CHE } 25 .^{14} \text { All these findings about the impact of RSBY on CHE were not significant. However, incidence of } \\
\text { CHE was significantly reduced for RSBY households with childbirth in last } 1 \text { year of data collection. }{ }^{25} \text { Two studies }{ }^{14} 19 \text { performed matching } \\
\text { and analysed using DID analysis, and other studies }{ }^{1625} \text { performed matching and linear and logistic regression. The cost of medicines was } \\
\text { significantly reduced by } 22 \text { INR for RSBY households in the rural areas; however, it increased for the urban households by } 28 \text { INR, but this } \\
\text { result was not significant. }{ }^{14}\end{array}$ \\
\hline
\end{tabular}

APL, Above poverty line; ATT, Average Treatment Effect on Treated; DID, Difference in Differences; NSSO, National Sample Survey Office ; OOPE, out-of-pocket expenditure; PSM, Propensity Score Matching; RSBY, Rashtriya Swasthya Bima Yojana.

For other PFHI schemes, the findings for effect of HI schemes on financial risk protection were mixed. Three studies reported a reduction in OOPE for insured households, ${ }^{20} 2126$ whereas another study reported no effect on OOPE. ${ }^{24}$ For households insured under VAS and RAS, no effect of these schemes was seen on OOPE. ${ }^{17}$ One study reported a reduction in in-patient drug expenditure for RAS households $;{ }^{15}$ however, other studies reported an increase in-patient household expenditure. ${ }^{27}{ }^{32}$ For CHIS in Tamil Nadu, one study reported no association of CHIS with size of OOPE ${ }^{17}$ and another study reported an increase in OOPE in-patient expenditure. ${ }^{33}$ It was reported that CHE was reduced for households enrolled under different
PFHI schemes, ${ }^{21}{ }^{28}$ however, specifically for VAS, one study reported reduction in $\mathrm{CHE},{ }^{31}$ and another study reported no association between CHE and insurance. ${ }^{17}$ For CHIS and RAS, no association was reported for CHE and insurance schemes. ${ }^{15} 17$ Enrolment in PMJAY did not decrease the OOPE or CHE of the enrolled households. ${ }^{29}$

Due to mixed evidence reported for the impact of PFHI schemes on different financial risk protection parameters, it is not possible to conclude whether these schemes have proven to be beneficial in reducing financial risk of the beneficiaries. A summary of these findings is given in tables 2 and 3 . 
Table 3 Impact of other public-funded health insurance (PFHI) schemes on financial risk protection and healthcare utilisation

Outcome
Expenditure (OOPE)

Catastrophic Health Expenditure (CHE)

\section{Findings}

The PFHI households were less likely to entail OOPE and there was a significant reduction in OOP for these households. ${ }^{20} 2126$ All the studies used regression analysis, linear and logit model for analysis. However, using Tobit regression, it was found that there was no effect of PFHI schemes on OOPE of the households. ${ }^{24}$ For Vajpayee Arogyashree Scheme (VAS), the OOPE was less for the insured households, when compared with uninsured households; however, the two-stage least squares (2sls) regression model reported no association between VAS enrolment and size of OOPE. ${ }^{17}$ According to Barnes et $a l,{ }^{31}$ reduction in OOPE increased with increase in quantiles of spending. At the 75th quantile, the significant reduction in OOPE for VAS households was Indian National Rupees (INR) 4485 , and at 95th quantile, it was INR 23548.19. There was no association between RAS (Andhra Pradesh- AP) enrolment and size of OOPE, by using 2sls regression model. ${ }^{17}$ By using difference-in-differences (DID), among phase 1 (2007), for Arogyashree enrolled households (AP), significant reduction in per-capital monthly OOP inpatient expenditure and inpatient drug expenditure was observed and an increase in inpatient expenditure for RAS households. ${ }^{27}$ For Rajiv Arogya Shree (RAS) (AP), Katyal et al ${ }^{32}$ reported a significant increase in both public and private inpatient expenditure, when calculated for the year 2004 and 2012 via DID analysis. Enrolment in CHIS of Tamil Nadu was not significantly associated with size of OOPE. ${ }^{17}$ For the CHIS operational in Kerala, the mean OOP expenses for inpatient services among insured participants (INR 448.95) was significantly higher than that of the uninsured households (INR 159.93), using Mann-Whitney U test. ${ }^{33}$ There was one study ${ }^{29}$ that reported findings on the effect of Pradhan Mantri Jan Arogya Yojana (PMJAY) on OOPE and CHE. It was reported that enrolment in PMJAY did not decrease the OOPE or CHE. There was statistically insignificant more reduction in OOPE for PMJAY enrolled households than other PFHI enrolled households. Statistical significant reduction in log of OOPE was marginally more for PMJAY-enrolled households than other PFHIs. OLS model was used for calculation of the abovementioned continuous outcome variable. As per the Probit model, there was a significant increase in $\mathrm{CHE} 25$ and $\mathrm{CHE} 40$ of PMJAY-enrolled households. But not for Propensity Score Matching (PSM) model, wherein reduction in OOPE for PMJAY and other PFHI was significant and CHE10 was not associated with PMJAY and PFHI enrolment according to any of the models. The naïve OLS model showed no association between the size of OOPE and enrolment under PMJAY or any PFHI schemes, these findings did not change under propensity score matching and Instrumental Variable (IV) models.

Six studies 151721222831 reported the effect of PFHI schemes on CHE. The PFHIs led to reduction in CHE; however, the effect was very small. ${ }^{21} 28$ With PSM, the PFHI-enrolled households were $13 \%$ less likely to experience $\mathrm{CHE} 10 \%$ and $6 \%$ less likely to experience CHE25. For the lowest three quintiles, this effect was even less pronounced as only $0.4 \%$ of PFHI households and $1 \%$ of PFHI households were likely to experience $\mathrm{CHE} 10$ and $\mathrm{CHE} 25 .^{21}$ There was a consistent increase in the catastrophic headcount threshold $40 \%$ of non-food expenditure for treatment, outpatient, inpatient and drugs. ${ }^{22}$ This increase was even reported in a long-term sample that is, households that have been enrolled in the PFHI schemes for a year. Two studies ${ }^{22}{ }^{28}$ used DID for analysis, whereas another used logistic regression ${ }^{21}$ for analysis. The VAS scheme had a limited effect on CHE; there was no association between enrolment in VAS and CHE25, CHE40 and CHE10, using two-step IV Probit model. ${ }^{17}$ In another study, ${ }^{31}$ the percentage of VAS households borrowing money for health reasons in the past 1 year was significantly lower than non-VAS households. According to Barnes et al, ${ }^{31}$ there was a marginal reduction in \% of $\mathrm{CHE}$ (both as \% of non-food expenditure and total expenditure) for VAS households than nonVAS households. This finding consists of both non-significant and significant results; however, reduction for $40 \%$ and $80 \%$ of $\mathrm{CHE}$ of the total non-food expenditure and $40 \%$ of $\mathrm{CHE}$ of the total expenditure was a significant finding. Additionally, money spent by VAS households on CHE was significantly lesser than non-VAS households. For RAS in Andhra Pradesh, there was no association between RAS enrolment and CHE25, CHE40, CHE10, by using two-step IV Probit model. ${ }^{17}$ There was no clear effect of Arogyashree enrolment on CHE. ${ }^{15}$ Enrolment in CHIS of Tamil Nadu was not significantly associated with CHE25, CHE40 and CHE10. ${ }^{17}$

Impoverishment

The PFHIs had a marginal effect on the reduction of impoverishment of households. ${ }^{2122}$ For the overall sample, the PFHIs led to marginal reduction in overall impoverishment and OOP impoverishment, ${ }^{22}$ for both short-term and long-term samples (more than a year). However, in the state fixed effect model for overall impoverishment, it was reported that the PFHI schemes had no effect on impoverishment. The state-fixed effect model was used because of the assumption that presence of different state health insurance (HI) schemes alter the findings, and this was analysed using regression analysis. ${ }^{22}$ There was no significant difference seen among Arogyashree-enrolled households in AP, compared with south India and all India sample on impoverishment and impoverishment due to OOPE. ${ }^{15}$

Utilisation of healthcare Two studies ${ }^{36} 37$ exclusively assessed impact of VAS on hospital utilisation rate. There was significant increase in utilisation of healthcare for all tertiary care facilities. The quasi-randomised study ${ }^{36}$ suggested significant increase in healthcare utilisation with respect to accessing healthcare for any symptoms with adjusted difference of $4.96 \%$. The increase in rate of hospitalisation in primary and tertiary care varied from $4.3 \%$ to $12.3 \%$, showing the significant change in healthcare utilisation after the implementation of VAS. The quasi-randomised study ${ }^{37}$ found significant increase in treatment-seeking behaviour for symptoms associated with cardiac conditions than for non-cardiac symptoms. Eligible households for VAS were $4.4 \%$ more likely to seek treatment than non-eligible households. The RAS was assessed by Katyal et al. ${ }^{32}$ The DID analysis suggested increase in healthcare utilisation in Andhra Pradesh and hospitalisation. ${ }^{27}$ The five studies ${ }^{202124} 2633$ assessed the impact of CHIS and other PFHIs and suggested an increase in inpatient and outpatient services. The matched cross-sectional study ${ }^{33}$ suggests significant increase in overall utilisation of inpatient services and non-significant results with respect to outpatient services among CHIS insured compared with uninsured. The multivariate analysis ${ }^{24}$ showed increased hospitalisation, hospitalisation for chronic conditions, hospitalisation among all age groups for PFHI households. It was also observed via Tobit regression model, being enrolled in PFHI was not significantly associated with length of stay during hospitalisation, contradictory to people with chronic illness. Though the association of $\mathrm{HI}$ with healthcare utilisation was high, inequality

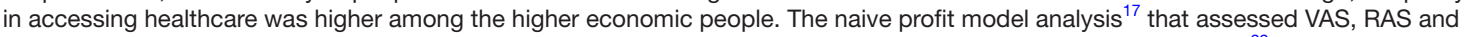
CHIS suggested significant increase in hospitalisation in Karnataka after the implementation of VAS. The only study ${ }^{29}$ that evaluated PMJAY; the data analysis from NSS data based on PSM and naive models on the hospitalisation did not show any significant difference in hospital care utilisation among both enrolled and non-enrolled population for insurance.

OLS, Ordinary Least Squares.

\section{Access and utilisation of health services}

Overall, 16 studies assessed the impact of PFHI on access and utilisation of health services (tables 2 and 3). The HI programmes were RSBY, ${ }^{14} 16232627303235$ VAS $^{36} 37$ RAS, ${ }^{172732}$ CHIS $^{20} 21242633$ and PMJAY. ${ }^{29}$ Of the 16 studies, 13 studies $^{14161720212324262729303637}$ were assessed to be of strong methodological quality, ${ }^{32}{ }^{33} 2$ were assessed as of moderate quality and ${ }^{35} 1$ was rated as weak quality. The analysis that was carried out majorly to look at the impact was logistic regression, profit models and other types. The outcomes that were reported include reporting of illness or morbidity, hospitalisation rate, outpatient care and in-patient care utilisation, duration of hospitalisation and utilisation of hospital services. Findings demonstrated increased access, utilisation of healthcare (both in rural and urban areas) and hospitalisation for RSBY. $^{1416232627303235}$ For other PFHI schemes like VAS, RAS 
and CHIS, an increase in utilisation of healthcare and in-patient outpatient services was reported. ${ }^{20} 21242632333637$ No significant difference in healthcare utilisation was reported for PMJAY beneficiaries. ${ }^{29}$

\section{Willingness-to-pay}

A high methodological study ${ }^{38}$ reported WTP for the insurance scheme. A majority (71 per cent) of CGHS beneficiaries considered that their current contribution was low and were willing to contribute more. Only 28 per cent Ex-servicemen Contributory Health Scheme beneficiaries were willing to pay an additional monthly financial contribution for better quality healthcare under the schemes. In comparison to higher employment grade beneficiaries, the CGHS beneficiaries from low employment grade were more willing to pay an additional amount to the existing monthly financial contribution.

\section{DISCUSSION}

This review identified and provided information on the impact of different PFHI schemes (operational in India) on healthcare utilisation, WTP and financial risk protection of the beneficiaries. It was observed that although the utilisation of healthcare services via in-patient and outpatient visits increased for insured beneficiaries, there was inconclusive evidence on the impact of different PFHII schemes on financial risk protection.

Our findings report that there is no conclusive evidence to suggest that RSBY reduced the OOPE and CHE or had an impact on financial risk protection. For other PFHIs including the state-sponsored PFHIs, viz., RAS, VAS and CHIS, the findings suggest a mixed impact of these schemes on OOPE, CHE and impoverishment, leading to inconclusive evidence for financial risk protection. Our findings are similar to another systematic review, ${ }^{10}$ which reported lack of substantial evidence for reduction in OOPE or improvement in financial risk protection by PFHI schemes in India.

For financial risk protection, varying results, from different studies for the same PFHI scheme, resulted in mixed findings for this outcome. Therefore, it was a challenge to pool evidence together and conclude on the impact of PFHI schemes on financial risk protection. One of the plausible reasons for this can be the different study designs and analysis methods used by different studies to assess the impact of financial risk protection. Also, difference in benefits packages and implementation of the scheme by various successive governments might have resulted in these mixed findings for this outcome.

One of the reasons for studies reporting no substantial impact of RSBY on financial risk protection can be the limited insurance cover, for example, INR 30000 annually under RSBY. As the utilisation of healthcare and hospitalisation under RSBY has increased over the years, ${ }^{10}$ it is possible that beneficiaries would have been hospitalised for hospital services of more than INR 30 000, leading to additional OOP payment. Hospitalisation for services not offered by the RSBY package and denial of hospitalisation by the empaneled hospitals has also led to an increase in OOPE. ${ }^{39}$ Another reason for the negligible impact of RSBY in reducing OOPE, as reported in some of the studies, can be the operational or functional error of the scheme. An important component of the scheme is the insurance companies, which are responsible for enrolling beneficiaries, empaneling hospitals, processing claims and reimbursing money. Delayed reimbursement from the insurance companies leads to hospitals asking beneficiaries to buy medicines and other consumables from outside, which results in high OOPE. Additionally, as there is no incentive for the insurance companies to keep a check on the OOPE payments, hospitals might charge patients or deny reimbursement of money on trivial grounds, leading to high OOPE. ${ }^{39}$ Another reason could be (which is based on personal experience of authors) to get an appointment for the surgery in empenelled hospitals, beneficiaries of the PFHIs usually wait for a longer period of time. Therefore, to avoid the delay in treatment, beneficiaries have to resort to OOP.

The impact of PFHIs (other than RSBY) including the statesponsored schemes was reported to be mixed and inconclusive, similar to another systematic review that reported lack of substantial evidence of impact on OOPE for PFHI operational in low and middle-income countries (LMICs). ${ }^{40}$ Additionally, as the functioning of any PFHI scheme depends on the governance, different governance structures and demographic profiles of the states would have led to heterogeneity in results. Poor impact of different PFHIs on financial risk protection (reported in some of the studies) can be attributed to similar factors that affect RSBY, that is, low coverage or benefits offered by the schemes leading to OOPE and CHE even for insured beneficiaries and interference or reimbursement issues due to functioning of insurance companies or 'trusts'.

This systematic review is the first one that has focused on the impact of PMJAY. Our findings suggest that there is a lack of evidence related to the impact of PMJAY, as only one study reported the poor impact of PMJAY on reduction in OOPE and financial risk protection. The reasons for poor impact can be similar as experienced by the earlier PFHIs schemes that is, problem of 'double billing', private providers monopoly and administrative problems. As PMJAY is a relatively new scheme, more evidence is needed to conclude on its impact. Additionally, as the only study included in the review was specifically for the state of Chhattisgarh, availability of evidence from other states is needed to summarise the impact of this scheme.

According to our review, there was an increase in incidence of outpatient and in-patient visits and the utilisation of medical services, however, the healthcare utilisation rate differed between states. The utilisation rate increased both among rural and urban areas for the RSBY and VAS. However, there was one study that assessed healthcare utilisation for PMJAY, and the results reported no significant increase in utilisation of healthcare by the PMJAY enrolees. One plausible reason for these results could be the lack of awareness regarding PMJAY, as it is a relatively new scheme. It is not justified to conclude based on a single study, and at the 
same time, it is important to look into various other aspects, due to which the results of the PMJAY are insignificant in increasing healthcare utilisation. The healthcare utilisation rate was assessed in terms of reporting morbidity, hospitalisation, utilisation of inpatient and outpatient services.

Overall, majority of the evidence suggests that implementation of PFHI has increased hospitalisation and the utilisation of outpatient care. Our findings are consistent with other systematic reviews, ${ }^{10} 40$ that is, PFHIs had a positive influence on utilisation of healthcare and hospitalisation in India and other LMICs. Although there is substantial evidence on the impact of PFHI on healthcare utilisation, more rigorous evaluation studies are required to evaluate the impact of health insurance schemes and especially the newly launched PMJAY.

It was reported that although the participants were willing to pay more, the findings for WTP are inconclusive, because the evidence is generated from a single study and the focus of the insurance was limited.

\section{Strengths and limitations}

Our review is the first comprehensive review, which has summarised the impact of PFHI schemes in India (including the new scheme of PMJAY under the Ayushman Bharat) on utilisation of healthcare and financial risk protection. One of the limitations of the review is the choice of quality assessment tool used for critical appraisal of included studies due to absence of any other valid tool for secondary data analysis. Responses to some of the questions and individual domain ratings for the EPHPP tool were subjective, although, before finalising the rating, we had a substantial discussion on every domain rating score. Additionally, the tool is used to assess quality of all the quantitative studies, which makes it very vague. Also, due to heterogeneity in methods, population and types of insurances, we could not perform meta-analysis.

\section{Implications of practice and research}

Our systematic review has vast policy and practice implications. Since UHC is one of the important components to achieve the sustainable development goals, the role of PFHI becomes even more important in providing equitable and affordable healthcare access to everyone. Financial risk protection is one of the key components of any PFHI scheme that ensures affordable healthcare for everyone. Poor impact of PFHIs on financial risk protection also indicates failure of the PFHI schemes. More research on PFHIs, especially PMJAY and its effect on financial risk protection and healthcare utilisation, are needed as this scheme is an important component of the Ayushman Bharat scheme under the UHC. Similarly, future studies can consider studying the effect of some of the state-funded insurances such as by the government of Goa and West Bengal, which also includes APL households, for which, currently, there is no evidence.

State and central governments could consider including APL households, especially middle-income group under the purview of PMJAY. There should be mechanisms to check corruption in the process of PFHI enrolment and focus could be provided to ease out the administrative difficulties faced by people at the time of claiming insurance. Future research in form of rigorous qualitative research, formative evaluations and process evaluations should be directed towards the reasons for the failure of different PFHIs in improving financial risk protection of the beneficiaries and demandside and supply-side barriers to implementation and uptake of PFHI. Research reporting reasons for failure of the PFHIs, in improving financial protection, will help in revising and modifying the functioning and implementation of the PFHI schemes for benefit of the consumers.

\section{CONCLUSION}

PFHI schemes, viz, RSBY, VAS, RAS and CHIS have been operational in India since 2008. These schemes have been impactful in increasing healthcare utilisation in terms of outpatient and in-patient care in both rural and urban areas. However, evidence related to financial risk protection was mixed and inconclusive. The new scheme of Pradhan Mantri Jan Arogya Yojana or PMJAY has incorporated administrative and strategic changes, which were based on the shortcomings of earlier PFHIs, viz., provision of a 24-hour inquiry helpline and increased coverage of healthcare services and benefit package. However, limited evidence available on the impact of PMJAY suggests no improvement in healthcare utilisation and financial risk protection of the beneficiaries. Future research on the impact of PMJAY and reasons for failure of other PFHIs on financial risk protection need to be explored.

\section{Author affiliations}

${ }^{1}$ Department of Health Information Management, Manipal College of Health Professions, Manipal Academy of Higher Education (MAHE), Manipal, Karnataka, India

${ }^{2}$ Department of Community Medicine, Kasturba Medical College, Mangalore, Manipal Academy of Higher Education, Manipal, Karnataka, India

${ }^{3}$ Department of Health Information, Public Health Evidence South Asia, Prasanna School of Public Health, Manipal Academy of Higher Education (MAHE), Manipal, Karnataka, India

${ }^{4}$ Manipal Institute of Communication, MAHE, Manipal, Karnataka, India

\section{Twitter Shradha S Parsekar @ParsekarShrads}

Acknowledgements We acknowledge PHRI-RESEARCH grant by Public Health Foundation of India, with the financial support of Department of Science and Technology to partially support authors to carry out this research. We would like to acknowledge the technical support provided by Public Health Evidence South Asia (PHESA), Prasanna School of Public Health (PSPH), Manipal Academy of Higher Education (MAHE), Manipal. We would like to thank Dr. Jisha B Krishnan, Research Assistant, PHESA, PSPH, MAHE, Manipal for supporting us in the title/abstract screening and quality assessment of the included studies and Dr. Vijay Shree Dhyani, Research Assistant, PHESA, PSPH, MAHE, Manipal, for supporting us in title abstract screening.

Contributors RB is the guarantor of the review. BTV, ER, RB and SSP conceptualised the topic. RV developed search strategy and conducted the search. SSP carried out title/abstract screening and BTV, ER, SSP carried out full text screening. BTV, ER and SSP extracted first round of data extraction, analysed and synthesised the data for the review. Extracted data from all the included studies was cross-checked and independent extraction was done for one third randomly selected studies by BTV, ER, SSP. Quality assessment was performed by BTV, ER, SSP. BTV, ER, SSP drafted the first version of report, which was further edited by RB, BTV, ER, RV, BU and SSP. All the authors read, provided feedback and approved the final report.

Funding The authors have not declared a specific grant for this research from any funding agency in the public, commercial or not-for-profit sectors.

Competing interests None declared. 
Patient consent for publication Not applicable.

Ethics approval This study does not involve human participants.

Provenance and peer review Not commissioned; externally peer reviewed.

Data availability statement Data are available upon reasonable request. All data relevant to the study are included in the article or uploaded as supplementary information. The datasets used and/or analysed during the current study are available from the corresponding author on request.

Supplemental material This content has been supplied by the author(s). It has not been vetted by BMJ Publishing Group Limited (BMJ) and may not have been peer-reviewed. Any opinions or recommendations discussed are solely those of the author(s) and are not endorsed by BMJ. BMJ disclaims all liability and responsibility arising from any reliance placed on the content. Where the content includes any translated material, BMJ does not warrant the accuracy and reliability of the translations (including but not limited to local regulations, clinical guidelines, terminology, drug names and drug dosages), and is not responsible for any error and/or omissions arising from translation and adaptation or otherwise.

Open access This is an open access article distributed in accordance with the Creative Commons Attribution Non Commercial (CC BY-NC 4.0) license, which permits others to distribute, remix, adapt, build upon this work non-commercially, and license their derivative works on different terms, provided the original work is properly cited, appropriate credit is given, any changes made indicated, and the use is non-commercial. See: http://creativecommons.org/licenses/by-nc/4.0/.

\section{ORCID iDs}

Shradha S Parsekar http://orcid.org/0000-0002-8824-9198

Bhumika Tumkur Venkatesh http://orcid.org/0000-0002-3338-6478

\section{REFERENCES}

1 Chokshi M, Patil B, Khanna R, et al. Health systems in India. J Perinatol 2016;36:S9-12.

2 Rao NV, Downey L, Jain N, et al. Priority-setting, the Indian way. J Glob Health 2018;8:020311-11.

3 Sengupta A, Nundy S. The private health sector in India. BMJ 2005;331:1157-8.

4 Balarajan Y, Selvaraj S, Subramanian SV. Health care and equity in India. The Lancet 2011;377:505-15.

5 Hooda SK. Out-of-pocket Payments for Healthcare in India:Who Have Affected the Most and Why? J Health Manag 2017;19:1-15.

6 Zodpey S, Farooqui HH. Universal health coverage in India: progress achieved \& the way forward. Indian J Med Res 2018;147:327-9.

7 The World Health Organization. Universal health coverage Geneva, 2020. Available: http://www.who.int/health_financing/universal coverage_definition/en/ [Accessed 23 June 2020].

8 Twelfth five year plan (2012-2017), 2013. New Delhi, India: planning Commission, government of India

9 Ayushman Bharat for a new India -2022, announced, 2018. Press information bureau government of India Ministry of finance

10 Prinja S, Chauhan AS, Karan A, et al. Impact of publicly financed health insurance schemes on healthcare utilization and financial risk protection in India: a systematic review. PLoS One 2017;12:e0170996.

11 Higgins JPT TJ, Chandler J, Cumpston M, eds. Cochrane Handbook for Systematic Reviews of Interventions version 6.1. Vol. 2020. Cochrane, September 2020.

12 Moher D, Liberati A, Tetzlaff J, et al. Preferred reporting items for systematic reviews and meta-analyses: the PRISMA statement. BMJ 2009;339:b2535

13 Effective public health practice Project- quality assessment tool for quantitative studies. Ontario: MacMaster university. Available: https:// merst.ca/ephpp/2020

14 Azam M. Does social health insurance reduce financial burden? panel data evidence from India. World Dev 2018;102:1-17.

15 Fan VY, Karan A, Mahal A. State health insurance and out-of-pocket health expenditures in Andhra Pradesh, India. Int J Health Care Finance Econ 2012;12:189-215.

16 Ghosh S, Gupta ND. Targeting and effects of Rashtriya Swasthya bimA Yojana on access to care and financial protection. Econ Polit Wkly 2017;52:61-70.

17 Garg S, Chowdhury S, Sundararaman T. Utilisation and financial protection for hospital care under publicly funded health insurance in three states in southern India. BMC Health Serv Res 2019;19:1004.
18 Johnson D, Krishnaswamy K. The impact of RSBY on hospital utilization and out-of-pocket health expenditure. Washington, DC: World Bank, 2012

19 Karan A, Yip W, Mahal A. Extending health insurance to the poor in India: an impact evaluation of Rashtriya Swasthya Bima Yojana on out of pocket spending for healthcare. Soc Sci Med 2017;181:83-92.

20 Mahapatro SR, Singh P, Singh Y. How effective health insurance schemes are in tackling economic burden of healthcare in India. Clinical Epidemiology and Global Health 2018;6:75-82.

21 Ranjan A, Dixit P, Mukhopadhyay I, et al. Effectiveness of government strategies for financial protection against costs of hospitalization care in India. BMC Public Health 2018;18:501.

22 Ravi S, Bergkvist S, National Council of Applied Economic Research. Are publicly financed health insurance schemes working in India? India Policy Forum 2015;11:158-92.

23 Raza W, van de Poel E, Panda P. Analyses of enrolment, dropout and effectiveness of RSBY in northern rural India. MPRA paper 70081 Germany: University Library of Munich, 2016.

24 Sriram S, Khan MM. Effect of health insurance program for the poor on out-of-pocket inpatient care cost in India: evidence from a nationally representative cross-sectional survey. BMC Health Serv Res 2020;20:839.

25 Sinha RK. Impact of publicly financed health insurance scheme (Rashtriya Swasthya bimA Yojana) from equity and efficiency perspectives. Vikalpa 2018;43:191-206.

26 Nandi S, Schneider H, Dixit P. Hospital utilization and out of pocket expenditure in public and private sectors under the universal government health insurance scheme in Chhattisgarh state, India: lessons for universal health coverage. PLoS One 2017;12:e0187904.

27 Rao M, Katyal A, Singh PV, et al. Changes in addressing inequalities in access to hospital care in Andhra Pradesh and Maharashtra states of India: a difference-in-differences study using repeated crosssectional surveys. BMJ Open 2014;4:e004471.

28 Selvaraj S, Karan AK. Why publicly-financed health insurance schemes are ineffective in providing financial risk protection. Economic and Political Weekly 2012;47:60-8.

29 Garg S, Bebarta KK, Tripathi N. Performance of India's national publicly funded health insurance scheme, Pradhan Mantri Jan Arogaya Yojana (PMJAY), in improving access and financial protection for hospital care: findings from household surveys in Chhattisgarh state. BMC Public Health 2020;20:949.

30 Sabharwal NS, Mishra VK, Naik AK. How does social protection contribute to social inclusion in India? Evidence from the Nationa health insurance programme (RSBY) in Maharashtra and Uttar Pradesh: overseas development Institute, 2014

31 Barnes K, Mukherji A, Mullen P, et al. Financial risk protection from social health insurance. J Health Econ 2017;55:14-29.

32 Katyal A, Singh PV, Bergkvist S, et al. Private sector participation in delivering tertiary health care: a dichotomy of access and affordability across two Indian states. Health Policy Plan 2015;30 Suppl 1:i23-31.

33 Philip NE, Kannan S, Sarma SP. Utilization of comprehensive health insurance scheme, Kerala: a comparative study of insured and uninsured Below-Poverty-Line households. Asia Pac J Public Health 2016;28:77s-85.

34 Khetrapal S, Acharya A. Expanding healthcare coverage: an experience from Rashtriya Swasthya Bima Yojna. Indian J Med Res 2019;149:369-75.

35 Dror DM, Vellakkal S. Is RSBY India's platform to implementing universal hospital insurance? Indian J Med Res 2012;135:56-63.

36 Sood N, Bendavid E, Mukherji A, et al. Government health insurance for people below poverty line in India: quasi-experimental evaluation of insurance and health outcomes. BMJ 2014;349:g5114.

37 Sood N, Wagner Z. Impact of health insurance for tertiary care on postoperative outcomes and seeking care for symptoms: quasi-experimental evidence from Karnataka, India. BMJ Open 2016;6:e010512.

38 Vellakkal S, Juyal S, Mehdi A. Healthcare Delivery and Stakeholder's Satisfaction Under Social Health Insurance Schemes in India: An Evaluation of Central Government Health Scheme (CGHS) and Exservicemen Contributory Health Scheme (ECHS). SSRN 2012;5.

39 Devadasan N, Seshadri T, Trivedi M, et al. Promoting universal financial protection: evidence from the Rashtriya Swasthya Bima Yojana (RSBY) in Gujarat, India. Health Res Policy Syst 2013;11:29.

40 Acharya A, Vellakkal S, Taylor F. Impact of national health insurance for the poor and the informal sector in low- and middle-income countries: a systematic review. London: University of London, Institute of Education, Social Science Research Unit, 2012. 